MATEC Web of Conferences 48,03005 (2016)

DOI: $10.1051 /$ matecconf $/ 20164803005$

(C) Owned by the authors, published by EDP Sciences, 2016

\title{
Ultrasonic tomography of complex shaped carbon fiber composites
}

\author{
Vadim Zhvyrblya ${ }^{1, a}$, German Filippov ${ }^{1}$, Elizaveta Sedanova ${ }^{1}$ and Yana Salchak $^{1}$ \\ ${ }^{1}$ National Research Tomsk Polytechnic University, 634050 Tomsk, Russia
}

\begin{abstract}
Non-destructive quality assurance of complex shaped objects is one of the major challenges in the variety of industrial areas. Robot-assisted ultrasound tomography is one of the common methods used for this purpose. This study proposes a tomography based on the preliminary received 3-D reconstruction results. The obtained experimental details represent the applicability of the suggested method to ensure effective quality control.
\end{abstract}

\section{Introduction}

The rapid development of aerospace industry caused commensurate growth in composite industry. The geometry of composite structures has become more complex, that, in its turn, caused limitations of automatic nondestructive testing procedures. The most convenient in this case are robotic systems of control that enable accurate positioning of the transducer in accordance with the shape of an object and its surface normal.

However, implementation of a robotic system of control requires relevant 3-D model of the controlled object and precise location of the object according to the coordinate system of the equipment. One of the ways to create a 3-D model is three-dimensional reconstruction based on structured light techniques.

\section{Digital Focus Array}

At present, ultrasound testing methods are usually based on the registration of the echo-signal scattered from the back wall and inner flaws. The most common method is that implies the application of a single-channel transducer. That kind of transducer consists of one transmitting element and one receiving element. These two elements can be combined into one as well. In that case single-channel transducer emits acoustic wave and then receives the scattered signal.

However, the approach of single-channel transducers has several disadvantages compared with multi-channel transducers. For example, it is more time consuming and does not provide accurate results of the measurement. One of the testing methods where multi-channel transducers (phased array transducer) are applied is Digital Focus Array (DFA) technique.

The difference between single-channel testing method and DFA is the procedure of obtaining the data. Digital Focus Array Technique is applied with only one phased array element sending out a

\footnotetext{
${ }^{a}$ Corresponding author : vzhrb3001@gmail.com
} 


\section{MATEC Web of Conferences}

signal, the rest elements are receivers. The signal can be considered as a wavelet that is generated by one individual element of the array. The phased array is moving on the fixed distance from controlled object during the inspection. Received time signals are saved and then form the matrix of units. This matrix is a basis for the further image reconstruction. The time of flight is evaluated for each unit. The necessary characteristics for image reconstruction are calculated according to the following correlation:

For the element with $x, y, z$ coordinates the image reconstruction parameter is:

$$
A(x, y, z)=\left|\sum_{x_{b}=x_{b 1}}^{x_{b} 2} \sum_{x_{c}=x_{c 1}}^{x_{c 2}} P_{e}\left(t_{b c}\right)\right|
$$

$\mathrm{A}(x, y, z)$ corresponds to the transparency of the image in $x, y, z$ coordinate system. The high frequency echo-signals are summed up. Index "e" corresponds to one of elements that generate signals: transmitter and receiver. The time is correlated with the point of the image $(x, y, z)$ in accordance with the location of ultrasonic transducers:

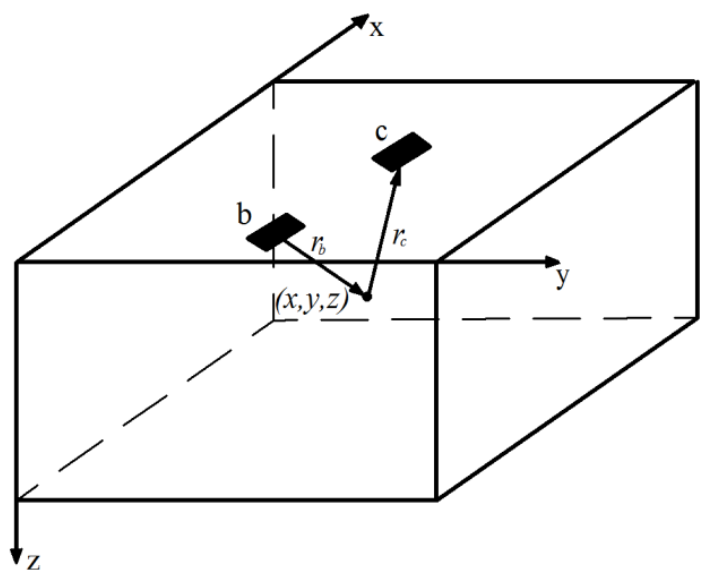

Figure 1. Image reconstruction principles.

$$
r_{b}=\sqrt{\left(x_{b}-x\right)^{2}+\left(y_{b}-y\right)^{2}+z^{2}}, r_{c}=\sqrt{\left(x_{c}-x\right)^{2}+\left(y_{c}-y\right)^{2}+z^{2}}
$$

In the correlation given above index "b" corresponds to the number of the transmitter and index "c" corresponds to the number of the receiver (see Fig. 1).

The following image reconstruction algorithm is proposed: the controlled area is divided into separate voxels with a given step. For each voxel of the reconstruction area the wave propagation time to and back from the element is calculated. Further the amplitude value of wave propagation time is summed up:

$$
A_{M}(x, y, z)=\sum_{i=1}^{n} A_{i}(x, y, z)
$$

In order to reduce noise in image reconstruction the following correlation is applied:

$$
\mathrm{A}_{\mathrm{M}}(x, y, z)=\sum_{\mathrm{i} \# \mathrm{j}} \mathrm{A}_{\mathrm{i}}(x, y, z) \cdot \mathrm{A}_{\mathrm{i}}(x, y, z)
$$

DFA technique enables an accurate determination of the defect location. The results of the inspection are represented as A-scans that display summarized amplitude values of the signals from 
each separate voxel in accordance with each element of the transducer. This in turn improves the sensitivity and the resolution in the near field of transducer. The resolution of the method depends on the length of the impulse, the size of the phased array and the ultrasound velocity.

The robot-assisted ultrasound tomography system was developed by Tomsk Open Laboratory for Material Inspection (TOLMI) of TPU for the implementation of DFA technique. The system consists of the scanner, the immersion tank, the measurement and control unit. One of the most important parameters that are necessary for the proper inspection is the scanner path. For that reason, it is vital to have the precise model of the controlled object. If the object has a simple geometry, then it is not difficult to generate its model by using CAD system. On the other hand, if the object instead of simple shape has a complex curved shape then modeling can become a difficult, time-consuming procedure.

The study suggests an implementation of optical reconstruction technique that provides a valid way to generate a model of the controlled object [1-3].

\section{Experimental Procedure}

The composite structure in the shape of a blade was selected for the experiment (see Fig.2). The surface of the sample was not linear and had irregular radial deformations over the whole area.

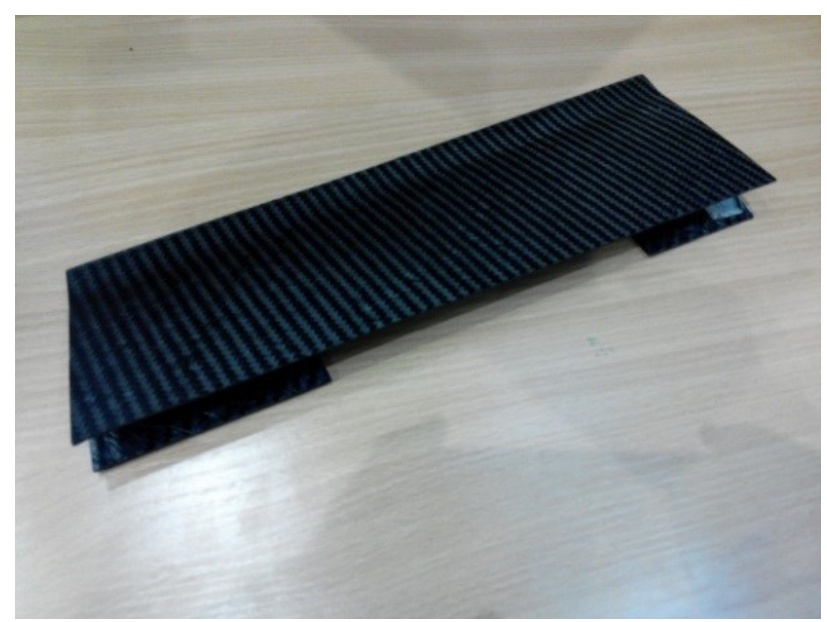

Figure 2. The object of control.

Artificial defects were performed in the object in order to evaluate the sensitivity of proposed method of control. There were drilled flat-bottom holes with diameters of 3, 6 and 9 millimeters respectively. Each of the defect was placed at a different depth (see Fig. 2).

A very accurate 3-D model of the controlled object surface is required for the correct setting of the scanner path. Three-dimensional reconstruction based on structured light techniques was chosen as approach for model creation. The experimental setup was designed for this purpose (see Fig.3.1). In order to improve the quality of 3-D reconstruction it was suggested to define a sector of the object by covering its surface with matte white coating.

The point cloud in accordance with the controlled object geometry is formed as a result of 3-D reconstruction. Deviation of the results does not exceed 150 micrometers due to the system's characteristics. 

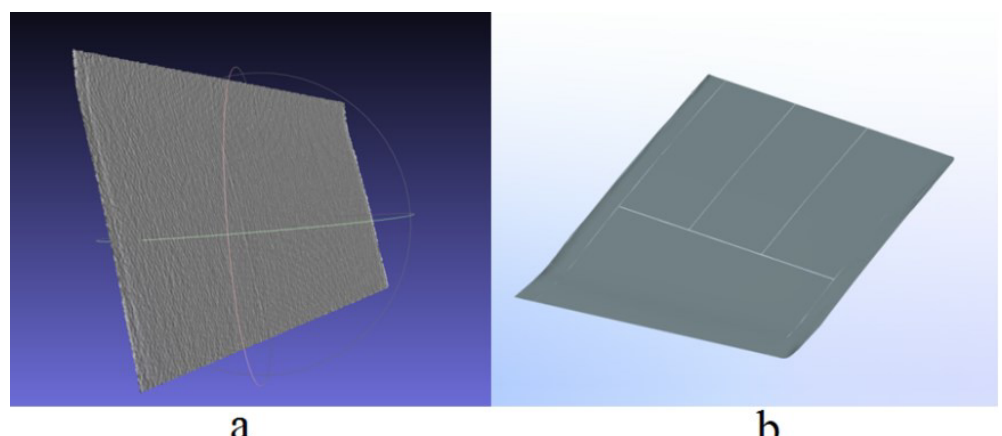

Figure 3. Point cloud $-\mathrm{a}$, surface $-\mathrm{b}$.

The algorithm of scanner path setting allows implementation of T-splines or NURBIS-surfaces. Thus, it is proposed to transform point cloud into NURBIS-surface.

The transformation procedure includes several steps:

1. Transformation of sparse point cloud into regular

2. Creation of the polygonal model by triangulation

3. Generation of the merged grid and its normalization

4. Division of the grid into T-splines

5. Transformation of T-splines into NURBIS-surfaces

Mathematical description of the NURBIS-surface obtainment could be found in [1].

The model of the controlled object was divided into sectors to give more efficient presentation of the results. There are three defects in each of the sectors. The defects are located at the different depth (see Fig.3a and Fig.3b)[4].

The experimental composite sample was scanned by the robotic system of ultrasonic testing. The single channel equipment with $15 \mathrm{MHz}$ transducer was applied.

The first step of the experiment is intercallibration of the scanner and the object of control, that can be performed by optical or tactile probing methods. The scanning path is generated by the means of specialized software in accordance with the surface that was preliminary generated.

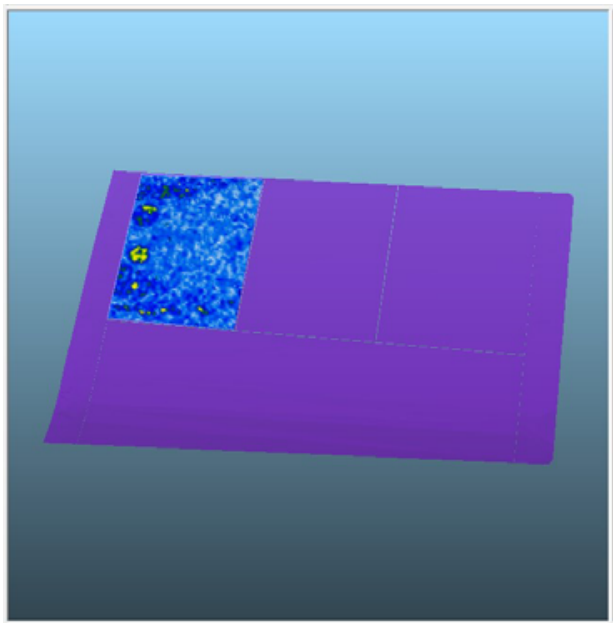

a

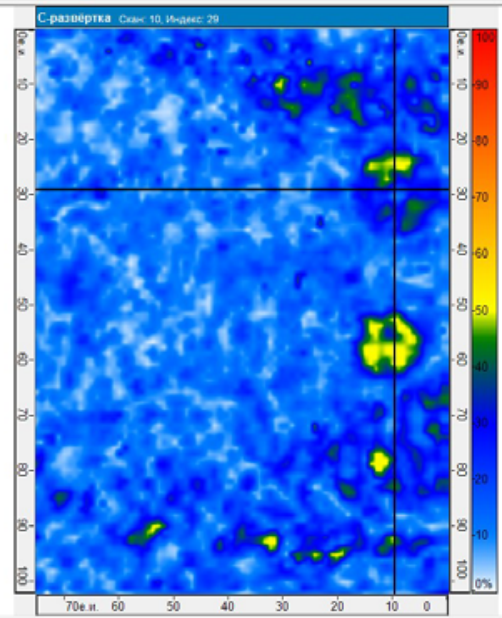

b

Figure 4. Reconstruction of the first sector-scan ( $\mathrm{a}-$ on the object, $\mathrm{b}-\mathrm{C}$-scan).

Further the scanner is placed into the work position with the surface of the controlled object. Sensitivity setting is arranged. The main parameters that should be set are the following: amplification gain and time corrected gain (TCG).

After that, an automatic procedure starts which enables further analysis of the obtained data. 
Additional noise and complex geometry of the bottom surface relief make it rather difficult to reconstruct all the defects at the same image. As noted earlier, this process could be simplified by division the object of control into several sectors. Therefore, the results for the defects located at the same depth are presented as individual sector-scans.

Figure $4 \mathrm{a}$ and $4 \mathrm{~b}$ demonstrates indications of ultrasound scans relevant to the defects with diameters of 3 and 6 millimeters. The defect with diameter of $9 \mathrm{~mm}$ is not quite noticeable due to the fact that its depth of occurrence is close to zero. This is caused by the rough bottom surface relief.

The indications of all three defects are defined very clearly at the second sector-scan. The level of the additional noise is rather slight (see Fig.5a and Fig.5b).



a

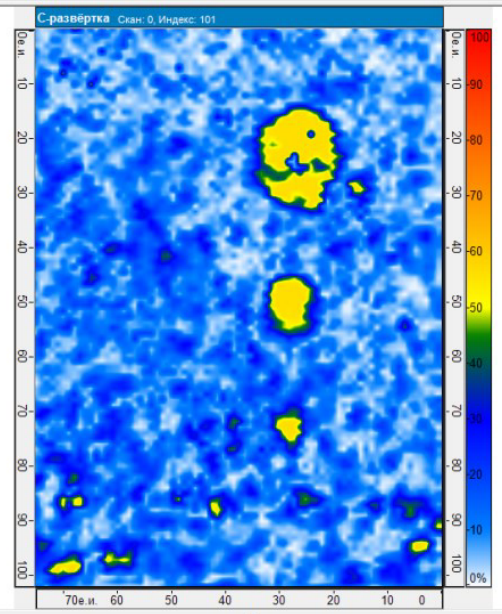

b

Figure 5. Reconstruction of the second sector-scan ( $\mathrm{a}-$ on the object, $\mathrm{b}-\mathrm{C}$-scan).

The defects of the third sector are located close to the surface and that causes superposition of defect indications and indications of surface reflected signals (see Fig.6a and Fig.6b). However, pulse transmission method allows to get clear indications of defects projections.

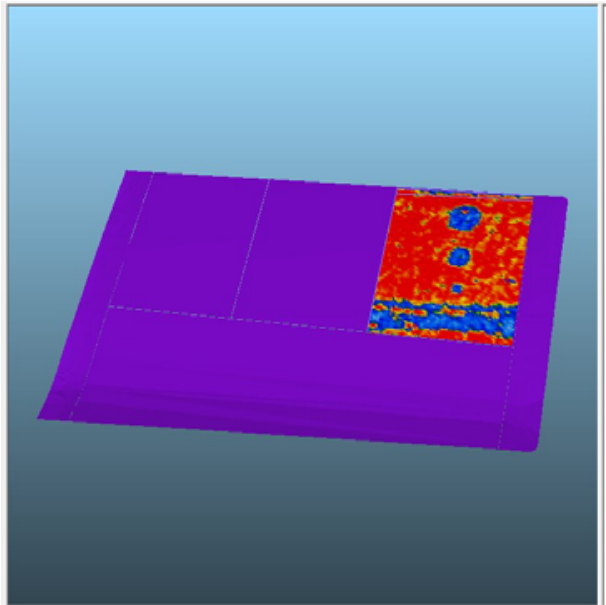

a



$\mathrm{b}$

Figure 6. Reconstruction of the third sector-scan ( $\mathrm{a}$ - on the object, b-C-scan). 


\section{MATEC Web of Conferences}

\section{Conclusion}

The accuracy of the inspection results can be affected not only by the parameters of the equipment and its calibration efficiency, but also by the scanner path. The scanner path is to be set exactly in line with the shape of the object to provide positioning of the transducers with multiple axis robotic manipulator. Due to the fact that the scanner path is formed according to the CAD-model of the object, the time that is needed for the model generation is important.

The implementation of optical reconstruction technique allowed to generate CAD-models of controlled objects with an accuracy of $150 \mu \mathrm{m}$. The obtained experimental results demonstrate that the proposed method of the surface model reconstruction provides effective nondestructive control of complex shaped carbon fiber composite materials. Moreover, within the results it is possible to identify 8 out of 9 defects indisputably.

\section{Acknowledgement}

The research was supported by Russian Governmental program "Science", research project No. 1524.

\section{References}

1. I. O. Bolotina, M. E. Djyakina, M. K. Krening, F. Mokhr, K. M. Reddi, A. I. Soldatov, E. Zhantlesov, Russ. J. Nondestruct. 49, 145 (2013)

2. D. Sednev, A. Lider, D. Demyanuk, M. Kroening, Y. Salchak, Phys.Procedia 70, 505 (2015)

3. M. Spies, W. Jager, Ultrasonics 41, 125 (2003)

4. D. Lanman, D. Crispell, G. Taubin, 3DIM 3, 107 (2007) 